\title{
Electronic structure of the $\mathrm{Sr}_{0.4} \mathrm{Ca}_{13.6} \mathrm{Cu}_{24} \mathrm{O}_{41}$ incommensurate compound
}

\author{
Alain Gellé, Marie-Bernadette Lepetit \\ Laboratoire de Physique Quantique, IRSAMC / UMR 5626, Université Paul Sabatier, \\ 118 route de Narbonne, F-31062 Toulouse Cedex 4, FRANCE
}

(Dated: November 21, 2018)

\begin{abstract}
We extracted, from strongly-correlated ab-initio calculations, a complete model for the chain subsystem of the $\mathrm{Sr}_{0.4} \mathrm{Ca}_{13.6} \mathrm{Cu}_{24} \mathrm{O}_{41}$ incommensurate compound. A second neighbor $t-J+V$ model has been determined as a function of the fourth crystallographic parameter $\tau$, for both low and room temperature crystallographic structures. The analysis of the obtained model shows the crucial importance of the structural modulations on the electronic structure through the on-site energies and the magnetic interactions. The structural distortions are characterized by their long range effect on the cited parameters that hinder the reliability of analyses such as BVS. One of the most striking results is the existence of antiferromagnetic nearest-neighbor interactions for metalligand-metal angles of $90^{\circ}$. A detailed analysis of the electron localization and spin arrangement is presented as a function of the chain to ladder hole transfer and of the temperature. The obtained spin arrangement is in agreement with antiferromagnetic correlations in the chain direction at low temperature.
\end{abstract}

PACS numbers: 71.10.Fd, 71.27.+a, 71.23.Ft

\section{INTRODUCTION}

One dimensional systems have attracted a lot of attention in the last decade. Indeed, they present specific properties due to the constrain imposed to electronic motion in two of the dimensions. One of their main characteristic is the nature of their low energy excitations that cannot be described in term of quasi particle as in the Fermi liquid theory. On one hand, a great deal of theoretical work have been done in order to predict the low energy physics of such systems ${ }^{1}$, in particular on chain and ladder systems ${ }^{2}$. On the other hand, solid state chemists have synthesized new compounds in order to test the theoretical predictions.

The so-called telephone number compounds, $\mathrm{Sr}_{14-x} \mathrm{Ca}_{x} \mathrm{Cu}_{24} \mathrm{O}_{41}$, are of special interest in this context since they present both chain and ladder subsystems ${ }^{3}$. For instance the $x=13.6$ compound presents under pressure a superconductive phase $e^{4}$ believed to be a possible realization of the theoretically predicted superconductivity in ladder systems ${ }^{\frac{5}{}}$. This compound, however, is quite intriguing since it does not present the expected properties, specific either of its chain or ladder subsystems. In particular, the isovalent substitution of strontium by calcium counter-ions totally modify the magnetic low temperature properties. Indeed, while the $x=0$ compound exhibits formation of second-neighbors spin dimers in the chain subsystem, these dimers disappear with increasing $\mathrm{Ca}$ content and an antiferromagnetic phase appears for highly doped systems.

The $\mathrm{Sr}_{14-x} \mathrm{Ca}_{x} \mathrm{Cu}_{24} \mathrm{O}_{41}$ compounds are layered systems composed of alternated slabs of spin chains and spin ladders. The chains and ladders run in the same direction, namely $\vec{c}$, however their respective unit cell parameter in this direction are incommensurate. The consequence of this "misfit" is a reciprocal distortion of the two subsystems under the influence of electrostatic and steric effects of the other. Indeed, each subsystems presents a structural incommensurate modulation with the periodicity of the other subsystem. This effect is specially important in the chain subsystem where the $\mathrm{Cu}$ $\mathrm{O}-\mathrm{Cu}$ angular distortion can reach $10^{\circ}$ from the nominal value of $90^{\circ}$ (from $88^{\circ}$ to $99^{\circ}$ ), and the copper-copper distances varies from $2.48 \AA$ up to $2.98 \AA$.

Unlike what was currently assumed, we recently showed - from ab-initio calculations - that these modulations are crucial in order to understand the low energy physics of these compounds ${ }^{6}$. The detailed study of the undoped compound ${ }^{7}$ revealed that its specific modulations are responsible for the electron localization observed in the chain subsystem, the formation of secondneighbor dimers and the existence of Zhang-Rice singlets. It has also been possible to explain the relative occurrence of free spins and dimers observed in the magnetic susceptibility measurements ${ }^{\underline{8}}$.

The present paper will present an extensive abinitio study of a highly calcium-doped phase, namely the $\mathrm{Sr}_{0.4} \mathrm{Ca}_{13.6} \mathrm{Cu}_{24} \mathrm{O}_{41}$ compound and a model of its chain subsystem taking explicitly into account the incommensurate modulations. Let us remind that the $\mathrm{Sr}_{14-x} \mathrm{Ca}_{x} \mathrm{Cu}_{24} \mathrm{O}_{41}$ systems have an intrinsic doping of 6 holes per formula unit (f.u.). The relative repartition of these holes between the chain and ladder subsystems is an opened question, that is believed to be of importance for the superconducting properties. The experimental results agree on the fact that an increasing part of the holes are located on the ladder subsystem as the calcium content increases. However, they disagree on the actual number of transferred holes. In the highly-doped compounds, the evaluations of the number of holes per f.u. on the ladders go from 1.1 hole in the X-ray experiments 9 up to 2.8 holes in optical conductivity experiments 10 . In addition, NMR ${ }^{11}$ experiments show an important varia- 
tion of the number of transferred holes as a function of the temperature. We will thus attach a special interest to this question in the present work.

The next section will be devoted to computational method, in section III we will present and analyze a second neighbor $t-J+V$ model Hamiltonian for the chain subsystem, extracted from the ab-initio calculations. This model has been determined using the crystallographic structures both at room temperature $\frac{12}{2}$ and at low temperature $(5 \mathrm{~K})^{13}$. Section IV will discuss the chain filling and hole transfer to the ladders as well as the magnetic order. Finally the last section will be devoted to the conclusion.

\section{THE AB-INITIO METHOD}

In strongly correlated systems, the magnetic and transfer interactions are essentially local. They can thus be evaluated using local but precise methods, able to treat strong correlation effects. This can be done, for instance, using embedded-fragment ab-initio spectroscopy methods $\frac{14}{4}$, that allow to take advantage of the quantum chemical multi-reference correlated codes. Indeed, we used in the present work the Difference-Dedicated Configuration Interaction method ${ }^{15}$ that is able to treat properly - within a finite system - (i) the open-shell character of the magnetic/hole orbitals, (ii) the strong electronic correlation of these orbitals, (iii) as well as the screening effects. The effects of the rest of the crystal on the selected fragment are treated through an appropriate bath of charges (Madelung potential) and total ion pseudo-potentials 16 (exclusion effect). Such approaches have been successfully used to study systems such as the high $T_{c}$ copper oxides $\frac{17}{}$ or the famous $\alpha^{\prime} \mathrm{NaV}_{2} \mathrm{O}_{5}$ compound 18 .

The quantum fragments are defined so that to include (i) the magnetic centers, (ii) the bridging oxygens mediating the interactions, and (iii) the first coordination shell of the preceding atoms which is responsible for the essential part of the screening effects ${ }^{14}$. First neighbor interactions are thus determined using $\mathrm{Cu}_{2} \mathrm{O}_{6}$ fragments (see figure 1 $\mathrm{k}$ ), while second-neighbor ones are computed using $\mathrm{Cu}_{3} \mathrm{O}_{8}$ fragments (see figure $1 \mathrm{~b}$ ). The different parameters of the $t-J+V$ model Hamiltonian are extracted from the ab initio calculations so that to reproduce both the computed excitation energies and the associated wave-functions, projected onto the magnetic orbitals. NN exchange, $J_{1}$, is directly given by the singlettriplet excitation energy when two magnetic electrons are considered in the small fragment. $\mathrm{NN}$ hopping, $t_{1}$, and magnetic orbital energy differences between adjacent sites, $\delta \varepsilon$, are extracted from the first two doublet states of the same fragment with one electron less. NNN exchange interactions, $J_{2}$, are extracted from the doublet-quartet excitations energies of the three-centers fragments with 3 magnetic electrons. NNN hopping and first neighbor bi-electronic Coulomb repulsion are obtained from the 3 singlets and 3 triplets of same fragment with one magnetic electron less. Let us point out that the 3 centers calculations also yield the NN interactions. The comparison between the evaluations of the first-neighbor integrals obtained from the 2 centers and 3 centers fragments allows us to verify the relevance of the chosen model and the fragment size dependence of our calculations. Some deviations can however occur since for the three centers fragments, the calculations where too large and we have been forced to restrain the set of treated screening excitations by deleting the most energetic ones. In practice, we have excluded from the calculations all excitations going from the deepest occupied atomic orbitals $(\varepsilon<-80 \mathrm{eV})$ and toward the most energetic unoccupied orbitals $(\varepsilon>80 \mathrm{eV})$.

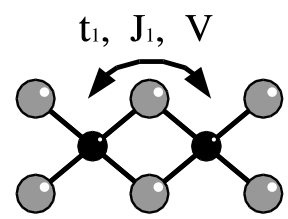

a) Two coppers fragment $\mathrm{Cu}_{2} \mathrm{O}_{6}$

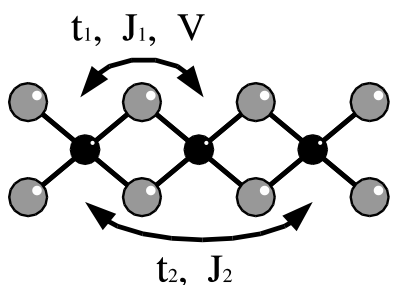

b) Three coppers fragment $\mathrm{Cu}_{3} \mathrm{O}_{8}$
FIG. 1: a) Schematic representation of the computed fragments. a) two centers b) three centers. The gray circles represent oxygen atoms, while the black circles represent the copper atoms.

A least-square fit method is finally used in order to extract the effective parameters from the ab-initio calculations.

Calculations $\frac{19}{}$ were done on 11 equivalent fragments located at 11 successive positions in the chain direction. These 11 fragments give a quite good representation of the different distortions occurring on the chain subsystem. In order to fully represent the whole chain subsystem, these 11 sets of results were extrapolated, using Fourier's series analysis, as a function of the crystallographic fourth coordinate $\tau$, associated with the system incommensurate modulations.

Let us notice that in a complete crystallographic description $^{20}$, each chain atom possesses a fourth fractional coordinate $\tau_{i}=\vec{r}_{i} \cdot \vec{k}=z_{i} c_{c} / c_{l}$, where $\vec{r}_{i}$ is the atom position, $c_{c}$ and $c_{l}$ the unit cell parameters of the chain and ladder subsystem in the $\vec{c}$ direction, $\vec{k}=\vec{c}_{c}^{\star} c_{c} / c_{l}$ is the chain modulation vector, $z_{i}$ is the fractional coordinate of the atom in the $\vec{c}$ direction. In the model Hamiltonian used in present work, $\tau$ corresponds to the fourth coordinate of the chain unit-cell copper atom. It is defined except for a constant. 


\section{A SECOND NEIGHBOR $t-J+V$ MODEL}

In the chain subsystem the spins are supported by the $3 d_{x z}$ orbitals of the copper atoms (the $x$ and $z$ axes being taken respectively parallel to the $\vec{a}$ and $\vec{c}$ crystallographic translation vectors). The model Hamiltonian is thus supported by these copper orbitals and taken as a second neighbor $t-J+V$ model. Indeed, the strongly correlated character of the $3 d$ orbitals exclude the possibility for the $3 d_{x z}$ orbitals to be doubly-occupied and thus suggest a $t-J+V$ model. At the same time, the nearly $90^{\circ} \mathrm{Cu}-\mathrm{O}-\mathrm{Cu}$ angle is responsible for a strong hindering of the super-exchange mechanism and thus of weak first neighbor interactions. Second neighbor interactions are thus competitive with the NN ones and must be taken explicitly into account.

\section{A. The Zhang-Rice singlets}

The first question we would like to address is the nature of the orbitals supporting the holes. Indeed, it has been suggested ${ }^{9}$ that the holes and the spins could be supported by different orbitals and form Zhang-Rice singlets $^{21}$. On the undoped compound $(x=0)$ we have been able to check this hypothesis and show that while the spins where essentially supported by the copper $3 d_{x z}$ orbitals, the holes where essentially supported by a coppercentered oxygen $2 p$ orbital with the same local symme$\operatorname{try}{ }^{7}$.

In the present compound we performed the same analysis for all the computed two-centers fragments and the results appear to be very similar. Indeed, while the magnetic orbitals supporting the spins have an average $71 \%$ copper $3 d_{x z}$ content and $29 \%$ oxygen $2 p$ content, the orbitals supporting the holes have an average $12 \%$ copper $3 d_{x z}$ content and $88 \%$ oxygen $2 p$ content. The variations around these values are weak with standard deviations of at most $3 \%$.

It is noticeable that the composition of the spin/hole orbitals is very stable, as a function of

- the structural modulation,

- the temperature,

- the calcium content.

This result is somewhat surprising, since one could have expected an influence, on the composition of the hole orbitals, of the variations of the $\mathrm{Cu}-\mathrm{O}$ distances and of the rotation of the $\mathrm{O}-\mathrm{Cu}-\mathrm{O}$ planes around the $\vec{c}$ axis. Indeed, on one hand, the $\mathrm{Cu}-\mathrm{O}$ distances present a relative variation range of $16 \%$ in the room temperature structure and of $19 \%$ in the low temperature structure. On the other hand, the orientations of the $\mathrm{O}-\mathrm{Cu}-\mathrm{O}$ planes around the $\vec{c}$ axis vary with a maximal amplitude of $30^{\circ}$. Both parameters are expected to strongly affect the overlap between the copper $d_{x z}$ orbital and the oxygen $2 p$ orbitals, however it seems that they do not affect the composition of both the spin- and hole-supporting orbitals.

\section{B. The fourth coordinate fit}

The eleven sets of computed results were fitted as a function of the fourth crystallographic coordinate $\tau$, using a Fourier series, according to the following expression

$$
a_{0}+\sum_{n} a_{n} \cos \left[2 \pi n\left(\tau-\varphi_{n}\right)\right]
$$

Only terms with a non negligible contribution to the series were retained.

The results are summarized in tables Ia for the $5 \mathrm{~K}$ structure and Ib for the $300 K$ calculations. Let us notice that the parameters of the present compound necessitate more harmonics to be fitted than for the $x=0$ compound. This can be attributed to the larger structural modulations caused by the chemical pressure induced by the $C a$ doping.

(a) \begin{tabular}{r|rrrrrr}
$5 K$ & $\varepsilon$ & $V$ & $t_{1}$ & $t_{2}$ & $J_{1}$ & \multicolumn{1}{c}{$J_{2}$} \\
\hline$a_{0}$ & 0 & 578 & 69.1 & 167.9 & 8.99 & -4.80 \\
$a_{1}$ & 1001 & & -132.3 & -61.6 & -1.38 & 2.51 \\
$a_{2}$ & 574 & & -25.9 & 24.7 & 13.70 & 0.87 \\
$a_{3}$ & & & 31.7 & -37.5 & 0.72 & 3.07 \\
$a_{4}$ & & & 9.8 & & -4.41 & -0.80 \\
$a_{5}$ & & & & & -2.31 & \\
& & & & & & \\
$\varphi_{1}$ & 0.251 & & 0.899 & 0.047 & 0.933 & 0.039 \\
$\varphi_{2}$ & 0.499 & & 0.147 & 0.797 & 0.148 & 0.041 \\
$\varphi_{3}$ & & & 0.396 & 0.213 & 0.175 & 0.551 \\
$\varphi_{4}$ & & & 0.141 & & 0.145 & 0.126 \\
$\varphi_{5}$ & & & & & 0.998 &
\end{tabular}

(b) \begin{tabular}{r|rrrrrr}
$300 K$ & $\varepsilon$ & $V$ & $t_{1}$ & $t_{2}$ & $J_{1}$ & \multicolumn{1}{c}{$J_{2}$} \\
\hline$a_{0}$ & 0 & 561 & 69.4 & 146.8 & 16.16 & -3.73 \\
$a_{1}$ & -1075 & & 86.3 & 61.5 & -6.94 & 2.78 \\
$a_{2}$ & -560 & & -72.9 & 18.8 & 1.70 & -1.18 \\
$a_{3}$ & 330 & & 6.7 & 18.6 & -4.96 & 1.71 \\
$a_{4}$ & & & 13.5 & 12.8 & -4.02 & 0.76 \\
$a_{5}$ & & & & & -2.28 & \\
& & & & & & \\
$\varphi_{1}$ & 0.761 & & 0.406 & 0.589 & 0.382 & 0.100 \\
$\varphi_{2}$ & 0.246 & & 0.648 & 0.760 & 0.684 & 0.255 \\
$\varphi_{3}$ & 0.584 & & 0.406 & 0.066 & 0.399 & 0.556 \\
$\varphi_{4}$ & & & 0.164 & 0.462 & 0.136 & 0.072 \\
$\varphi_{5}$ & & & & & 0.413 &
\end{tabular}

TABLE I: Analytic fit of the $t-J+V$ second neighbor model, a) for structure at $5 K$, b) for structure at $300 K$. All energies are given in $\mathrm{meV}$.

\section{The orbital energy}

The orbital energy differences between NN sites for both low and room temperature structures are reported in figures 2 and 2 b. The deduced orbital energies $(\varepsilon)$ are reported in figures 25 and 28. The variations of the orbital energies are very large and span a range of more than $2 \mathrm{eV}$ (respectively 2.4 and $2.8 \mathrm{eV}$ for the two structures). As previously mentioned, these variations are 
larger than any other interactions, and are thus responsible for the electron localization. The electron localization
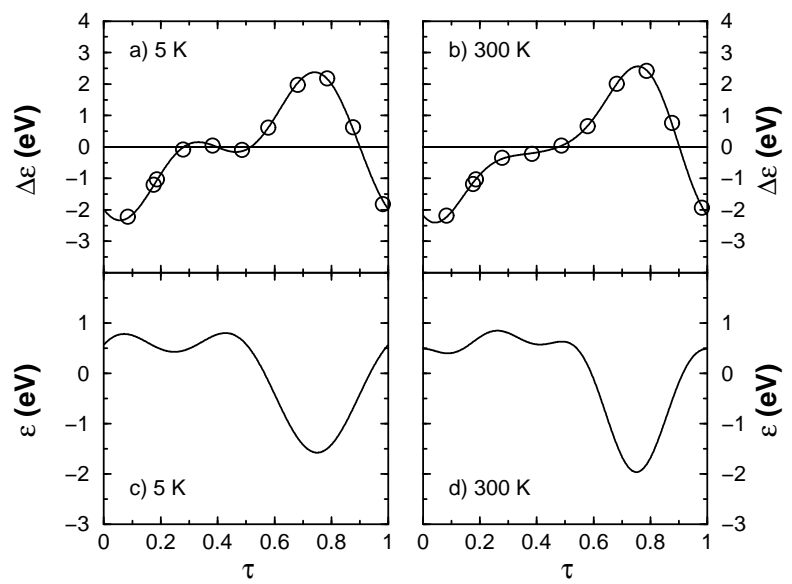

FIG. 2: a),b) Energy differences $(\delta \varepsilon)$ between two NN orbitals, respectively at $5 K$ and $300 K$, open circles correspond to computed values and solid line to the fit presented in table \ b),c) resulting orbital energies $(\varepsilon)$ respectively at $5 \mathrm{~K}$ and $300 \mathrm{~K}$.

can be clearly seen on the orbital energy curves since the latter present a large potential well near $\tau=0.75$. The widths of these well are respectively 0.45 and 0.4 for the $5 K$ and $300 K$ curves. One can thus think that about 4 electrons per f.u. will be strongly localized. Let us point out that these 4 electrons correspond to the nominal chain occupation if all the holes are located on them. Under the hypothesis that part of the holes are transferred to the ladders, the corresponding electrons transferred to the chains will be somewhat less localized due to low on-site orbital energies. Indeed, the rest of the potential curve is comparatively flat with an orbital energy variation of at most $0.5 \mathrm{eV}$, that is only twice as large as the hopping parameters.

Figure 3 reports the orbital energy differences between neighboring copper atoms as a function of the related Madelung potential differences. One see immediately, as was observed in the undoped compound, the nearly perfect proportionality relation (with a 0.41 slope) between the two. This relation shows the electrostatic origin of the orbital energy variations and the comparatively very weak influence of the other parameters. Let us however note that the most commonly-used tool to evaluate the oxidation state of the atoms and thus the site occupancy is not the Madelung potential evaluation but rather the Bond Valence Sum (BVS) method ${ }^{13.22}$. This empirical technique evaluates the oxidation state of a metal atom as a function of the distances $\left(r_{i j}\right)$ of the metal atom with its first coordination shell, that is

$$
\mathrm{BVS}_{i}=\sum_{j} \exp \left(\left(r_{o}-r_{i j}\right) / B\right)
$$

where $r_{0}$ and $B$ are parameters of the model. Figure 4

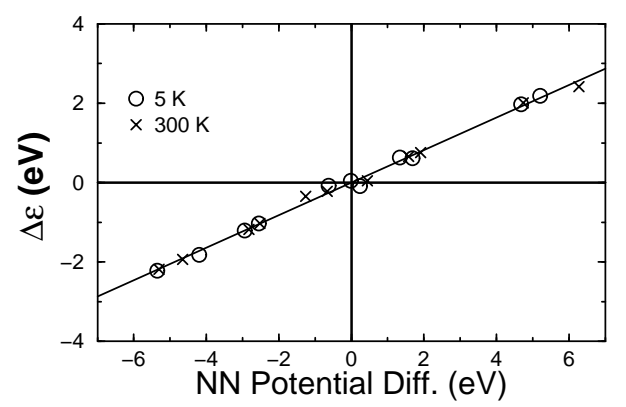

FIG. 3: Orbital energy differences between neighbor copper sites as a function of the related Madelung potential differences.

shows the Madelung potential and the BVS evaluations for the copper sites as a function of $\tau$. One sees immediately that while the large well present around $\tau=0.75$ in the Madelung potential and on-site energy curves is correctly seen by the BVS approximation, the energetic plateau seen for $\tau<0.5$ is not seen in the BVS curves. In fact, the BVS calculations exhibit another well around $\tau=0.25$. In the low temperature curve, the BVS thus sees a quasi-doubling of the modulation vector as in the $x=0$ compound. The use of these BVS results in order to predict the site filling leads to the existence of second neighbor dimeric units as in the undoped compound. Let us remind that the experimental results do not see any dimers but an antiferromagnetic order in this system. The failure of the BVS method to correctly predict the
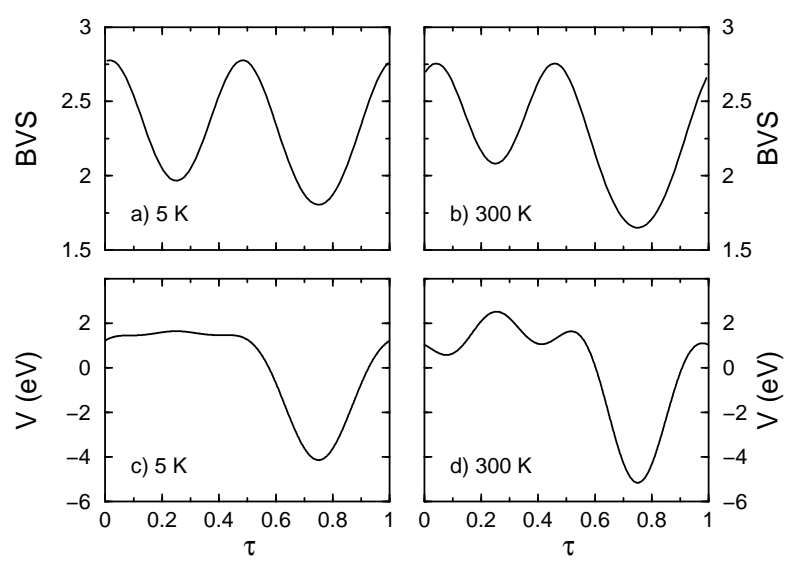

FIG. 4: Copper BVS values as a function of $\tau:$ a) $T=5 \mathrm{~K}$, b) $T=300 K$. Madelung potential on the copper sites : c) $T=5 K$, d) $T=300 K$. The BVS model parameters as been taken as in reference ${ }^{22}$, that is $r_{0}(C u-O)=1.679 \AA$, $B=0.37$.

electron localization in this system can be further analyze by detailing the contribution to the Madelung potential of a reference copper site, of the positions modulations of the other atoms up to a given distance. Figure 5 reports such an analysis for two adjacent sites (1 and 2), seen 
with opposite relative oxidations according whether it is computed with the BVS method or using the Madelung potential. One can make the following remarks.

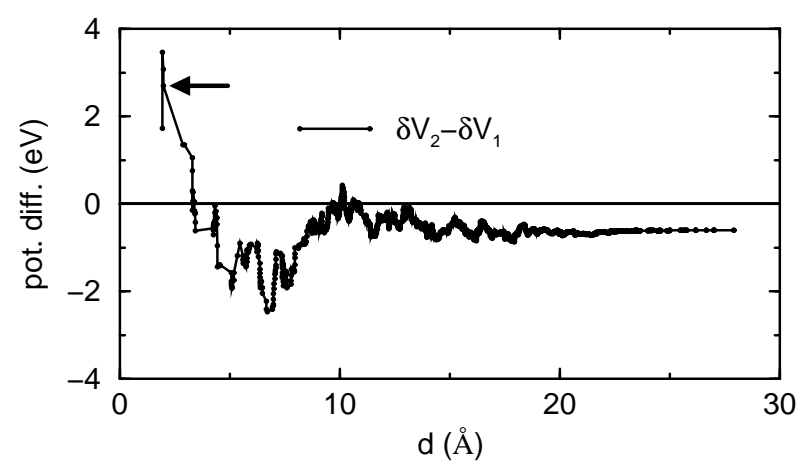

FIG. 5: Contribution of the structural modulation (modulated structure minus average structure) to the Madelung potential energy difference between adjacent sites, labeled as 1 and 2. The contribution is given as a function of the distances (to sites 1 and 2) up to which the modulations are taken into account. The dashed line represent the asymptotic limit.

- The potential difference between the two reference sites converge slowly. Indeed, in order to reach values that fluctuate around the correct asymptotic limit, one needs to include structural modulations up to at least $10 \AA$.

- When only the first coordination shells are considered (the four first neighbor oxygen atoms to each copper), the potential difference between the two copper 1 and 2 has the reverse sign compared to its asymptotic value, but the same one as the BVS. Let us remind that the BVS only take into account this first coordination shell. In fact, when the modulation is applied only on the first coordination shell, the Madelung potential and BVS methods yield similar results.

One can thus conclude that the origin the the BVS failure in the present system is due to the long range effect of the structural modulations on the electrostatic potential and thus on the on-site orbital energies.

\section{The first-neighbor interactions}

Figure 6 reports the NN hopping integrals as a function of the fourth crystallographic coordinate $\tau$. One sees immediately that, both in the low and room temperature phases, the hopping integrals present very large variations along the chain. Indeed, it ranges from $-60 \mathrm{meV}$ to $270 \mathrm{meV}$ in the low temperature phase and over a somewhat smaller range in the room temperature phase. Both phases present a large peak around $\tau=0.4$, however for $\tau \sim 0.9$ they exhibit quite different behaviors.

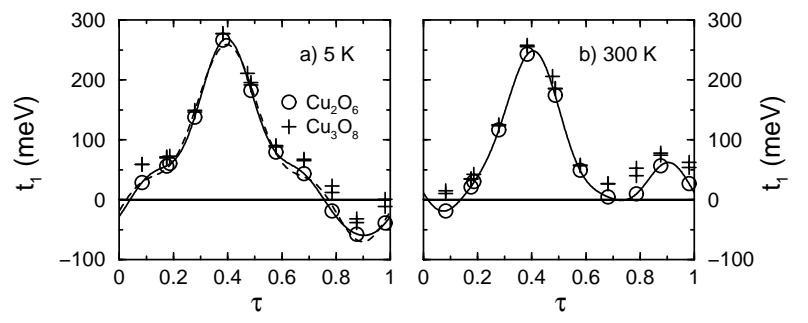

FIG. 6: NN hopping integrals as a function of $\tau$. a) $5 \mathrm{~K}$, b) $300 K$. The circles represent the computed values from the two copper fragments while the crosses represent the values computed from the three copper fragments. The solid line is the fit presented in table $\square$

Indeed, in the $5 \mathrm{~K}$ phase, the hopping integral presents a surprising behavior since it changes sign.

In order to explain these features, let us analyze the variation of the hopping integral as a function of different geometric parameters (see figure 7 ). The effective hopping between two magnetic orbitals can be expressed as the sum of a direct contribution or through-space contribution $\left(t_{d}\right)$ and a through-bridge contribution that goes via the bridging oxygens,

$$
t_{1}=t_{d}+\sum_{i} t_{i} t_{i}^{\prime} / \Delta_{i}
$$

where the sum runs over the different valence orbitals of the bridging atoms and $\Delta_{i}$ is the ligand-to-metal chargetransfer screened excitation energy. In the case of edgesharing $\mathrm{CuO}_{2}$ chains, the through-bridge contributions are in general negligible and the only important term is the through-space one. Indeed, the $90^{\circ} \mathrm{Cu}-\mathrm{O}-\mathrm{Cu}$ angles are responsible for destructive quantum interferences as far as the in-plane oxygen $2 p$ orbitals are concerned (further referred as $2 p_{\sigma}$ ). The other $2 p$ orbitals (referred as $2 p_{\pi}$ ), perpendicular to the chain plane, are orthogonal to the magnetic orbitals and thus the associated $\mathrm{Cu}-\mathrm{O}$ hopping terms (see figure $8 \mathrm{~b}$ ), $t_{\pi}$, are strictly zero. Finally, the $2 s$ orbitals are responsible for a contribution which is usually very small, due to the weakness of their overlap with the magnetic orbitals at the typical $\mathrm{Cu}-\mathrm{O}$ distances.

Taking now into account distortions from the ideal geometry, one generally considers that the leading perturbative term arises from the through-bridge contribution of the in-plane oxygen $2 p_{\sigma}$ orbitals. Indeed, this contribution is known to scale as $\cos (a) \simeq a-\pi / 2$, where $a$ is the $\mathrm{Cu}-\mathrm{O}-\mathrm{Cu}$ angle as defined in figure $8 \mathrm{k}$. Figure $7 \mathrm{k}$ and 77 reports the variations of the $\mathrm{Cu}-\mathrm{O}-\mathrm{Cu}$ angle for the presently studied compound. One sees immediately that the $t_{1}$ peak around $\tau=0.4$ is totally correlated with the strong increase of the $\mathrm{Cu}-\mathrm{O}-\mathrm{Cu}$ angle at both temperatures. Figure 8 shows the mechanism responsible for the increase of the effective hopping, $t_{1}$, through the increase of the $t_{\sigma}^{\prime}$ hopping term. For angles larger than $90^{\circ}$, as in the present case, $t_{\sigma}$ and $t_{\sigma}^{\prime}$ have the same sign and thus contribute positively to $t_{1}$. This mechanism explains the 
$t_{1}$ peak around $\tau=0.4$, however it cannot account for the negative peak observed in the low temperature phases around $\tau=0.9$.
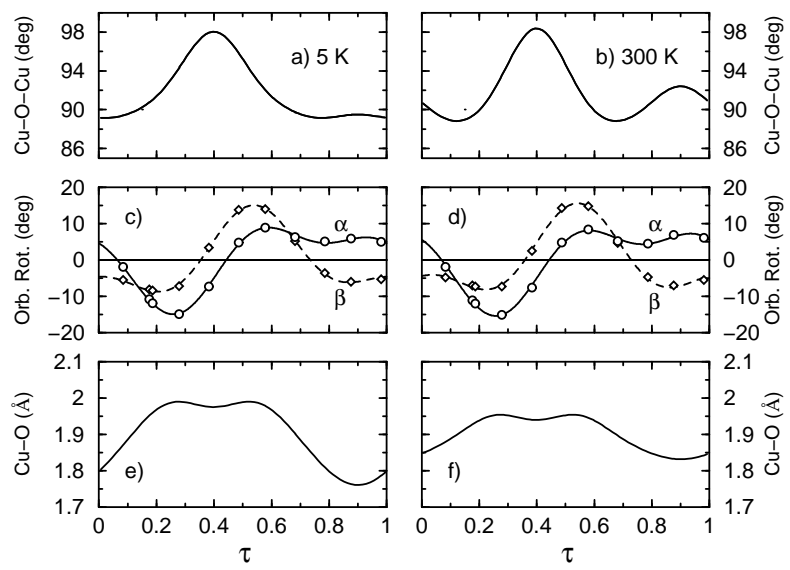

FIG. 7: a) and b) $\mathrm{Cu}-\mathrm{O}-\mathrm{Cu}$ angles . c) and d) Rotation angle of the magnetic orbital around the $\vec{c}$ axis, the reference being taken as the $\mathrm{Cu}-\mathrm{O}_{2}$ plane e) and f) average distance between two NN $\mathrm{Cu}$ atoms and their two bridging-oxygen ligands.

The second effect one can think of, is the set in of the $2 p_{\pi}$ orbital contribution. Indeed, the torsion of the $\mathrm{Cu}-$ $\mathrm{O}_{2}$ local planes compared to the reference average chain plane is responsible for a non zero $t_{\pi}$ term. More precisely, if $\alpha$ is the angle between the magnetic orbital and the $\mathrm{Cu}-\mathrm{O}_{2}$ plane, $t_{\pi}$ scales as $\sin (\alpha)$. Figures $7 \mathrm{~b}$ and 7 . report the variations of such $\alpha$ and associated $\beta$ torsion angles (see figure 80 for the definitions) for the studied compound. One sees immediately that unlike what was expected, there is not any correlation between the $\alpha$ and $\beta$ angles modulations and the low temperature peak around $\tau=0.9$. First, both low and room temperature structure exhibit very similar variations of $\alpha$ and $\beta$. Second, $\alpha$ and $\beta$ are of opposite signs in the $\tau=0.9$ region and thus induce a positive (and not the wanted negative) contribution to $t_{1}$.

The left over possible contribution is the $2 s$ one. This contribution, always active and of negative sign, is usually weak in amplitude due to the too large $\mathrm{Cu}-\mathrm{O}$ distances. Indeed, it scales exponentially with the $\mathrm{Cu}-\mathrm{O}$ distances. In the present case, it can be seen in figure 7 that the $\mathrm{Cu}-\mathrm{O}$ distances are very short around $\tau=0.9$. Let us notice that this distortion is much weaker in the room temperature phase and its effect is compensated by the $\mathrm{Cu}-\mathrm{O}-\mathrm{Cu}$ angle distortion that acts on $t_{1}$ with the opposite sign. In the low temperature structure the $\mathrm{Cu}-\mathrm{O}-\mathrm{Cu}$ angle stays close to $90^{\circ}$ (around $\tau=0.9$ ), thus the through-bridge contribution is essentially due to the $2 s$ orbitals which act negatively on $t_{1}$ and are thus responsible for the change in the hopping sign.

Figure 9] shows the variations of the NN exchange integral at both temperatures. One can observe that, as for the hopping, the exchange variations are very large compared to its average value. Indeed, the interaction even a)

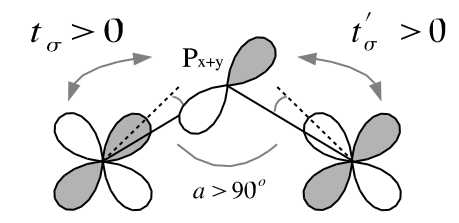

b)

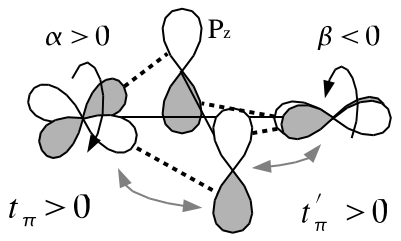

c)

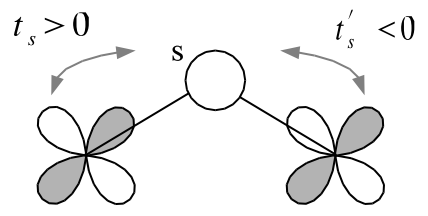

FIG. 8: Schematic contribution of the different throughbridge mechanism. a) through the oxygen $p_{\sigma}$ orbitals, b) through the oxygen $2 p_{\pi}$ orbitals, c) through the oxygen $2 s$ orbitals.

goes from ferromagnetic to antiferromagnetic both in the low and room temperature cases. As for the hopping, the effective exchange $J_{1}$ can be expressed as the sum of a direct or through-space contribution $J_{d}$ (always ferromagnetic in nature), an Anderson super-exchange term involving the direct hopping interactions, and throughbridge super exchange terms associated with the three oxygen orbital types,

$$
J_{1}=J_{d}-4 \frac{t_{d}^{2}}{U}-4 \sum_{i} \frac{t_{i}^{2} t_{i}^{2}}{\Delta_{i}^{2} U}
$$

where $U$ is the screened one-site coulombic repulsion on the magnetic orbital. As expected from equation [3 a strong correlation between the $t_{1}$ variations and the $J_{1}$ variations is observed in figure 9] Indeed, when the geometric distortions set in, the effective exchange integral starts to be dominated by the through-bridge mechanisms terms and becomes antiferromagnetic. This is the case around $\tau=0.4$ at both temperatures - due to the through-bridge super-exchange mediated by the $2 p_{\sigma}$ oxygen orbitals - and for $\tau=0.9$ in the low temperature case - due to the contribution of the oxygen $2 s$ orbitals.

This existence of chain regions for which the NN exchange is antiferromagnetic is, by itself, quite remarkable since it is always assumed that NN exchange is ferromagnetic in nature in such chain systems. In the low temperature case it corresponds to a non negligible part of the chain, namely $27 \%$. Let us also remind that such a phenomenon has not been observed in the undoped compound ${ }^{7}$ and should thus be put into perspective with the observation of antiferromagnetic correlations ${ }^{23,24,25}$ 
at low temperature in the calcium highly doped phases. This question will be discussed in somewhat more details in the next section.

Another important remark is the existence of antiferromagnetic NN exchange for $\mathrm{Cu}-\mathrm{O}-\mathrm{Cu}$ angle values very close to $90^{\circ}$ (in the $\tau \sim 0.9$ range). This goes against all assumed models of the magnetic interactions through $90^{\circ}$ angles 26 . Indeed, the role of the $2 s$ orbitals is usually ignored and this is the first case to our knowledge that their effect is not only non-negligible but dominating the interactions.

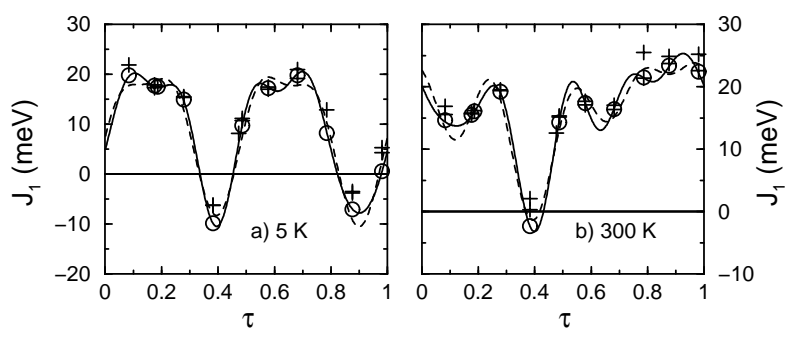

FIG. 9: NN exchange integrals as a function of $\tau$. a) $5 K$, b) $300 K$. The circles represent the computed values from the two copper fragments while the crosses represent the values computed from the three copper fragments. The solid line is the fit presented in table

\section{E. The second-neighbor interactions}

Figure 10 displays the effective hopping between second neighbor magnetic orbitals. As for the $\mathrm{Sr}_{14} \mathrm{Cu}_{24} \mathrm{O}_{41}$ undoped compound, the $t_{2}$ hopping integral is very large with an average value of $167 \mathrm{meV}$ at $5 \mathrm{~K}$ and $147 \mathrm{meV}$ at $300 \mathrm{~K}$. The variations are large, but unlike the NN hopping $t_{2}$ does not change sign. Indeed, in this case the dominating process is clearly the through-bridge process mediated by the $2 p_{\sigma}$ orbitals of the four bridging oxygen atoms. Figure 11 reports the second neighbor exchange
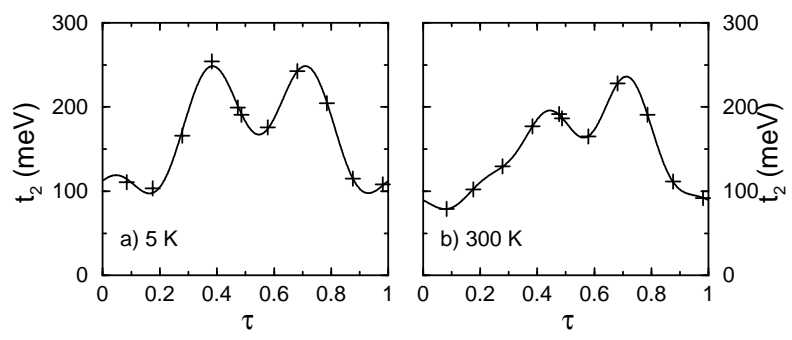

FIG. 10: Second neighbor hopping integrals as a function of $\tau$. a) $5 K$, b) $300 K$. The crosses represent the computed values, the solid line represent the fit.

integrals between the magnetic orbitals. As expected they are antiferromagnetic, the super-exchange mechanism being mediated by the same $2 p_{\sigma}$ orbitals as for the
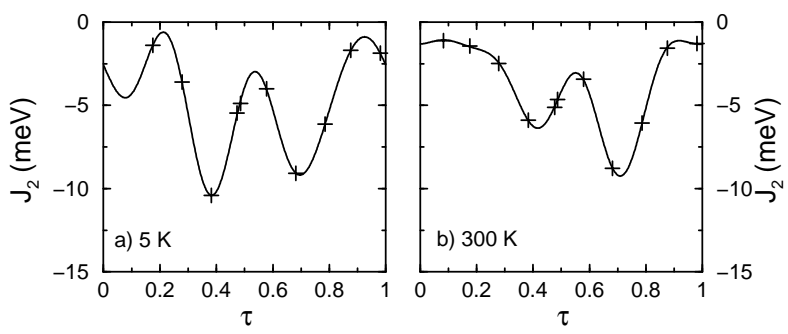

FIG. 11: Second neighbor exchange integrals as a function of $\tau$. a) $5 K$, b) $300 K$. The crosses represent the computed values, the solid line represent the fit.

hopping. Consequently, the maximal amplitude of $J_{2}$ is obtained for the largest values of $t_{2}$, as can be seen on figure 11

\section{FILLING ANALYSIS}

Let us now consider the possible fillings and associated electron localization in the chain subsystem. Figure 12 , displays the localization of the electrons over 77 consecutive sites along the chain, in the $5 \mathrm{~K}$ structure. The electrons are localized according to an energetic criterion taking into account both the on-site orbital energies and the NN bi-electronic repulsion. In order to see more clearly the effect of the bi-electronic repulsion we have reproduced the filling obtained without the NN repulsion as published in reference $\underline{\underline{6}}^{\underline{\underline{a}}}$ (figure). Spins are arranged according to NN and NNN exchange interactions. Three types of filling are considered in the range proposed in the literature, namely with one, two and three holes per f.u. transferred to the ladders.

One sees immediately that there is not any dimers for either of the fillings. The second important remark, is the presence at $5 \mathrm{~K}$ and for all fillings, of NN occupied sites with antiferromagnetic (AF) exchange. Indeed, the $\mathrm{AF}$ interactions allows the formation of clusters with no spin frustration. The $27 \%$ of $J_{1}$ antiferromagnetic values are thus directly related with the possibility to transfer electrons to the chain subsystem without increasing the magnetic energy. As can be seen from the comparison between figures [12b and 12r. the effects of the NN repulsions are (i) to increase the typical size of the spin clusters, (ii) to prevent the appearance of spin frustration in the clusters, while some can be observed when repulsions are not taken into account, (iii) to favor the disappearance of the free spins. Indeed, the latter disappear for $n \leq 2$, while $n \leq 3$ is necessary if the repulsion is not considered. Let us notice that free spins are not experimentally observed in this highly doped compound ${ }^{24}$, unlike the undoped one ${ }^{8}$.

In the room temperature phase, the formation of high spin cluster, partially frustrated is observed. Indeed, the small range of $\tau$ where the $\mathrm{NN}$ exchange integral is an- 
a ) $300 \mathrm{~K}$

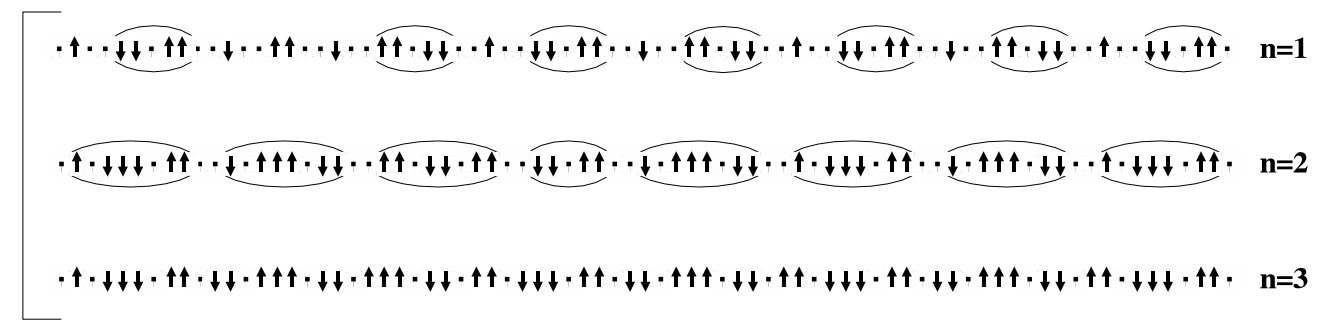

b ) $5 \mathbf{K}$

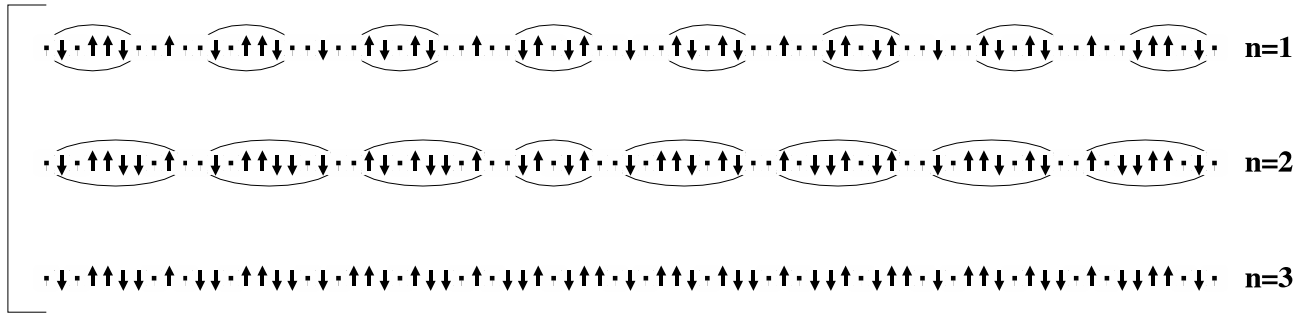

c) $5 \mathrm{~K}$

without

repulsion

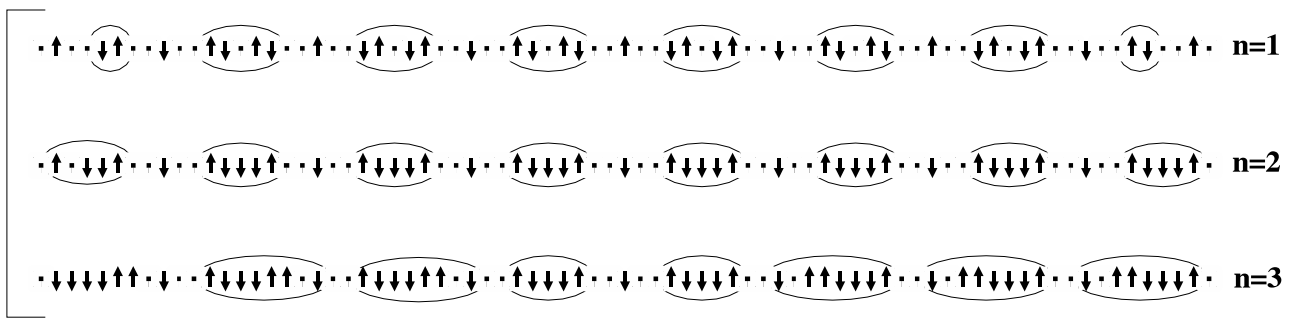

FIG. 12: Tentative electron localization as a function of the filling ( $n=1,2,3$ where $n$ is the number of holes per f.u. transferred to the ladders). a) and b) at $5 \mathrm{~K}$, c) at $300 \mathrm{~K}$. In the a) diagrams the localization is computed using the magnetic orbitals on-site energies only, in the b) and c) diagrams the electron localization is computed taking into account the NN coulombic repulsion in addition to the on-site energies.

tiferromagnetic is associated with consecutive sites supporting at least one hole. The consequence is that unlike what happens at $5 K$, the AF exchange parameter region is in this case non relevant for the physics.

\section{CONCLUSION}

The present paper proposes a second neighbor $t-J+V$ model for the $\mathrm{Sr}_{0.4} \mathrm{Ca}_{13.6} \mathrm{Cu}_{24} \mathrm{O}_{41}$ compound, as a function of the fourth crystallographic coordinate $\tau$. The model Hamiltonian has been extracted from a series of ab-initio calculations and is given both for the $5 \mathrm{~K}$ and $300 K$ structures.

The present study shows the crucial importance of the structural modulations on the low energy properties of the compound. Indeed, the on-site energy of the magnetic orbitals is strongly modulated by the atomic displacements and is affected by geometric variations occurring at distances up to $\sim 16 \AA$. The consequence of such long range dependence of the on-site energies is the non pertinence of methods such as BVS for the evaluation of the copper oxidation state and thus of the electron localization. The other important effect of the structural modulations is the appearance of large ranges of $\tau$ for which the NN exchange become antiferromagnetic despite the nearly $90^{\circ} \mathrm{Cu}-\mathrm{O}-\mathrm{Cu}$ angles. In particular, it has been observed for the first time dominant through-bridge exchange mechanism going via the oxygen $2 s$ orbital.

The consequence of these particularities on the low energy properties of this compound is a strong electron localization for a large part of the sites, and the disappearance of spin frustration in the low temperature phase. Another consequence is the incredible sensibility of the electronic structure and more specifically of the localization of the electrons to the specific distortions of the geometric structure.

One of the main remaining question in this family of systems and in particular in the calcium doped phases is the position of the Fermi level or in other words the relative chemical potential of the chains and ladders subsystems that drive the hole transfer between the latter. In order to answer such a question it is necessary to study the ladder-chain interactions, the relative positions of the on-site energies of both subsystems, the hopping between the two as well as the chain-ladder magnetic interactions that could be responsible for a three dimensional spin ordering. In particular, it would be interesting to know 
whether there exist large hopping terms between ladder and chain copper sites with on-site energies both near the Fermi level. The underlying question is to which extend these systems should be considered as quasi onedimensional.

Acknowledgment : the author would like to thank
Dr. J. Etrillard for discussions on the structural aspects, Dr. D. Maynau for providing us with the CASDI chain of programs, Dr. D. Poilblanc for helpful discussions. The calculations were performed at IDRIS/CNRS computational facilities under project number 1104 .
1 For a review see: Th. Giamarchi, Quantum Physics in one dimension, Clarendon Press, Oxford (2004), and references therein.

2 For reviews see : E. Dagotto and T.M. Rice, Science 271, 618 (1996) ; S. Maekawa, Science 273, 1515 (1996) ; B. Levy, Phys. Today, October 1996, page 17 ; T. M. Rice, Z. Phys. B 103, 165 (1997).

3 M. McCarron et al., Mater Res. Bull. 23, 1355 (1988)

4 M. Uehara et al., J. Phys. Soc. Jap. 65, 2764 (1996) ; T. Nagata et al., Phys. Rev. Lett. 81, 1090 (1998).

${ }^{5}$ E. Dagotto, J. Riera and D. Scalapino, Phys. Rev. B 45, 5744 (1992) ; M.Sigrist, T. M. Rice and F. C. Zhang, Phys. Rev. B 49, 12058 (1994) ; H. Tsunetsugu, M. Troyer and T. M. Rice, Phys. Rev. B 49, 16078 (1994).

6 A. Gellé and M.-B. Lepetit, Phys. Rev. Letters 92, 236402 (2004).

7 A. Gell and M.-B. Lepetit, unpublished.

8 S.A. Carter et al., Phys. Rev. Lett. 77, 1378 (1996).

${ }^{9}$ N. Nücker et al., Phys. Rev. B 62, 14384 (2000).

10 T. Osafune et al., Phys. Rev. Lett. 78, 1980 (1997).

11 K. R. Thurber, K. M. Shen, A. W. Hunt, T. Imai and F. C. Chou, Phys. Rev. B 67, 94512 (2003).

12 T. Ohta, F. Izumi, M. Onoda, M. Isobe, E. TakayamaMuromachi and A. W. Hewat, J. Phys. Soc. Jpn 66, 3107 (1997).

13 M. Isobe, M. Onoda, T. Ohta, F. Izumi, K. Kimoto and E. Takayama-Muromachi, Phys. Rev. B 62, 11667 (2000).

14 M.-B. Lepetit, Recent Research Develepments in Quantum Chemistry, p. 143, Vol. 3, Transworld research Network (2002).
15 J. Miralles, J. P. Daudey and R. Caballol, Chem. Phys. Lett. 198, 555 (1992) ; V. M. García et al., Chem. Phys. Lett. 238, 222 (1995) ; V. M. García, M. Reguero and R. Caballol, Theor. Chem. Acc. 98, 50 (1997).

16 N. W. Winter, R. M. Pitzer and D. K. Temple, J. Chem. Phys. 86, 3549 (1987).

17 D. Muñoz, F. Illas and I. de P.R. Moreira, Phys. Rev. Letters 84, 1579 (2000) ; D. Muñoz, I. de P.R. Moreira and F. Illas, Phys. Rev.B 65, 224521 (2002).

18 N. Suaud and M.-B. Lepetit, Phys. Rev. B 62402 (2000) ; N. Suaud and M.-B. Lepetit, Phys. Rev. Letters 88, 056405 (2002).

19 The calculations have been performed using the CASDI suite of programs provided of D. Maynau. The basis set and core effective potentials used can be found in : Z Barandiaran and L. Seijo, Can. J. Chem. 70, 409 (1992).

20 P.M. de Wolff, Acta Cryst. A 30, 777 (1974).

21 F. C. Zhang and T. M. Rice, Phys. Rev. B 37, 3759 (1988).

22 M. Isobe and E. Takayama-Muromachi, J. Phys. Soc. Jpn 67, 3119 (1998).

23 S. Ohsugi, K. Magishi, S. Matsumoto, Y. Kitaoka, T. Nagata and J. Akimitsu, Phys. Rev. Letters 82, 4715 (1999).

24 M. Isobe, Y. Uchida, and E. Takayama-Muromachi, Phys. Rev. B 59, 8703 (1999).

25 V. Kataev, K.-Y. Choi, M. Grüninger, U. Ammerhal, B. Büchner, A. Freimuth, and A, Recovleschi, Phys. Rev. B 64, 104422 (2001).

26 J. B. Goodenough, Magnetism and the Chemical Bond, Interscience, Wiley, New-York (1963). 